\title{
Taxonomy of Piscian Cestodes of Freshwater fish: A cladistic model of Morphotaxonomic Study of Rare Tapeworm from the Dehradun Region (U.K.), India
}

\author{
Amar Jeet Singh, Aditya Narayan, Sarvjeet Singh
}

\begin{abstract}
The current experimental study has been conducted to analyze cladistic taxonomic aspects of the piscian cestode parasites including its morphological characters. Freshwater fishes caught on the spot at selected sampling stations with the help of local fishermen and collected for tapeworm investigations. Freshwater catfish Clarias batrachus (Linnaeus, 1758) ${ }^{[3]}$ collected from Dehradun sampling stations and examined for tapeworm parasites as per experimental design. The collected fishes were brought to the Parasitology laboratory for experimental study and morphological as well as anatomical facts investigation. Parasites obtained from infected host fishes kept intact in 5\% formalin for further investigation. Ten cestode (tapeworm) parasites were found from its infested Intestine. The morphological study of tapeworms indicates that parasitic diversity is accessible within the Ganga River at Dehradun (U.K.) region. Morphotaxonomic characterization reported as - $\mathrm{H}$, butterfly flap shaped ovary, testes numerous and well developed, vitelline follicle - cortical and medullary. This study provides an overview of the piscian tapeworm parasites of some freshwater fishes inhabiting the Ganga River.
\end{abstract}

Keywords: Morphotaxonomic, Morphometry, Tapeworm, Cestode, Ganga River, Dehradun (U.K.).

\section{INTRODUCTION}

F ish provides the essential nutrients for the human body mainly in the form of micronutrients as well as macronutrients as complex proteins, lipids, vitamins, and minerals. Fish matter an important source of income and economic issues for such people who are related in fisheries, aquaculture and fish trade. The government of India conducts many research projects and training schemes from time to time, to improve fish quality and growing supply with fulfilled nutritional rich fishes [1]. Edible fishes are found

Revised Manuscript Received on April 20, 2020.

* Correspondence Author

Amar Jeet Singh, Department of Zoology, Institute of Basic Science, Bundelkhand University, Jhansi (U.P.), India. Email: amarjeet7321@gmail.com

Aditya Narayan, Department of Zoology, Institute of Basic Science, Bundelkhand University, Jhansi (U.P.), India. Email: adityanarayan21@gmail.com

Sarvjeet Singh, Department of NST, DTU, Delhi, India. Email: sarvjeetsingh779@gmail.com

(c) The Authors. Published by Blue Eyes Intelligence Engineering and Sciences Publication (BEIESP). This is an open access article under the CC BY-NC-ND license (http://creativecommons.org/licenses/by-nc-nd/4.0/) infested by tapeworm parasites which reduce the nutritional and economic value of infected fish because tapeworm parasites consume required nutrition from the host and secrete harmful substances. Due to this, the physical weight and size of fishes reduce and ultimately lose their market value [2]. Keeping all these facts in focus the morphotaxonomic study of tapeworm parasites is an urgent necessity today. Very little exploration has been done on the taxonomy of tapeworm parasites hence more experimental work is required. Much research has shown that the generic helminth parasites are found as cestode (tapeworm) parasites in freshwater edible fishes. The curiosity of the authors to develop understanding about the tapeworm parasites found in freshwater fishes lead them to undertake this project at different sampling stations. In the inclusion of this research study, the authors have limited restricted themselves to the taxonomy of cestode parasites of infected host fishes from various sampling stations of Ganga riverside at the Dehradun region. An analysis has been carried out incorporating all the taxonomic aspects and statistical parameters involved in the research (Table I, II).

\section{MATERIAL \& METHOD}

The methodology has been descended in this study with these following steps as an experimental protocol -

1. Sampling (Field Work)

a) Sampling from water bodies (Including local ponds, reservoirs, rivers and water tanks, etc.)

b)Sampling from local fish markets (Including village and township based street market)

2. Collection of fishes and rearing in water tanks/pots

3. Dissection

a) Virtual Dissection (Computer-based practice)

b) Handmade dissection at spot/at the sampling site (Experimental framework)

4. Removal of parasites from host fish (Collection of parasites)

5. Preservation of parasites

6. Permanent slides preparation in parasitology laboratory

7. Micrographs/Camera Lucida of prepared slides

8. Identification and observation of parasites (with morphological, anatomical and morphometrically aspects) 
The freshwater catfish namely Clarias batrachus (Linnaeus, 1758) ${ }^{[3]}$ collected from various sampling stations of Ganga riverside at the Dehradun region of Uttarakhand state for the morphotaxonomic study of piscian tapeworms.

Freshwater local fishes angled to caught on the spot at sampling stations with the help of local fishermen, collected and brought to the lab for tapeworms investigation. Fishes were dissected to checkout internal organs, the alimentary canal and various organs of the infected host were removed and cut open in $0.9 \%(\mathrm{w} / \mathrm{v})$ aqueous isotonic saline water in the petri dish. It was shaken several times and many of the associated waste materials were removed. The intestine, gills, stomach and body cavity of parasites were examined thoroughly under a dissecting microscope to ensure that no parasites are left behind. During the autopsy, scolex of parasites were attached deeply embedded in the fish body, which was taken out by scrapping the mucosa of the intestine. It requires a sharp-edged needle and scalpel to remove it carefully. After this, the segment of the mucosa and tissues attached to the parasite was removed by shaking the body of worm in saline water. The stretched and torsion worms were replaced in lukewarm saline water till changing in normal status. In the case of longer worms, these were lifted and repeated several times with the help of needles against the edges of the Petri dish and later on fixed in 5\% formalin. Harris' hematoxylin solution and eosin were used to stain whole worm specimen, finally cleared in xylene and mounted in Canada balsam as per experimental protocol. Camera lucida drawings were made using stage micrometer with an ocular micrometer. All the measurements $(\mathrm{L} \times \mathrm{W})$ were measured in millimeters. For calculation of bio-statistical parameters as X1, X2, Y1 and Y2 values, Margolis equations adopted [4].

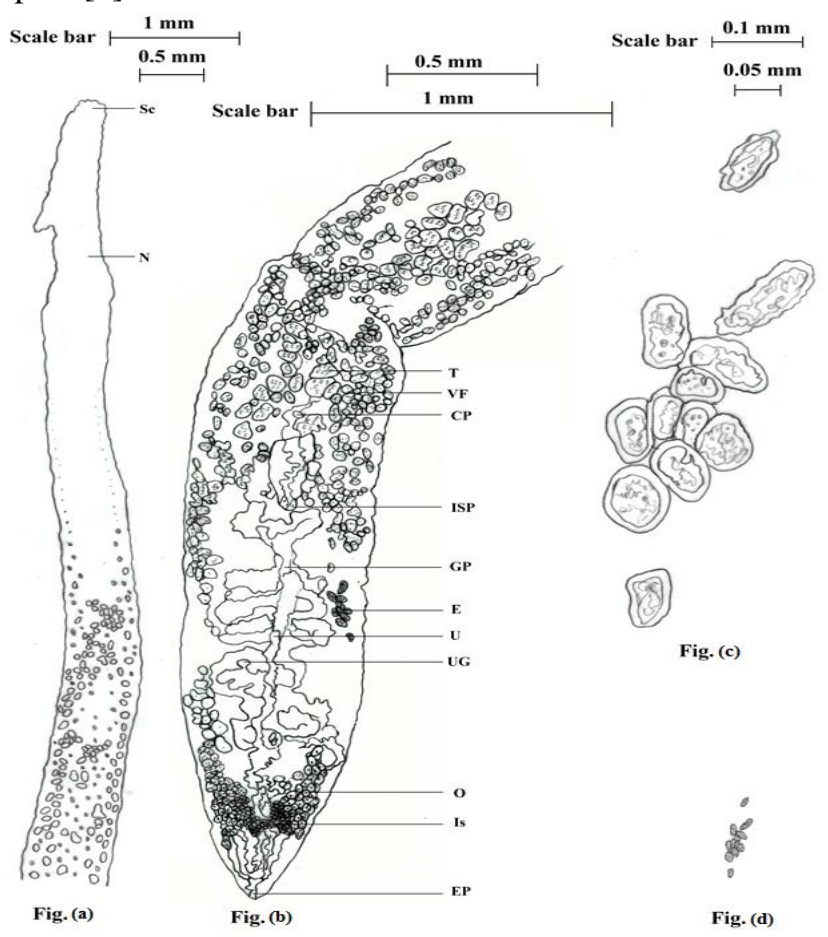

Fig. 1. (a) The anterior part of the worm with smooth scolex and neck [100x] (b) Middle and posterior part of the worm [100x] (c) Eggs [400x] (d) Eggs (bilobed \& ovular) $[100 x]$

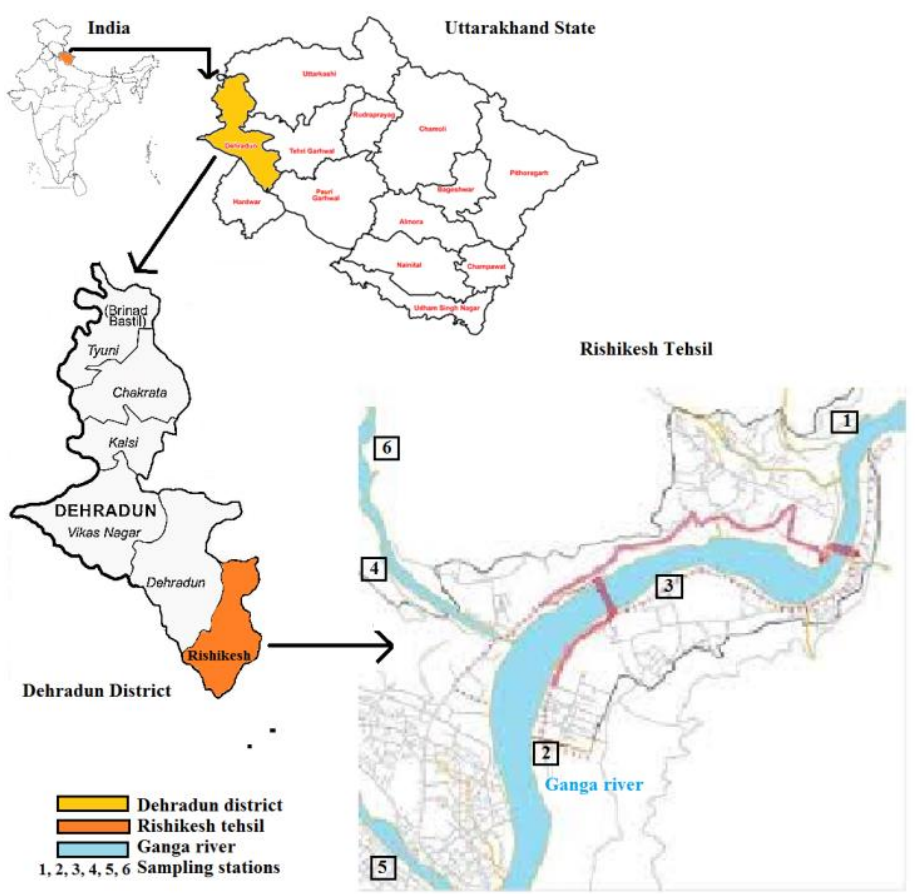

Fig. 2. Map showing of Ganga river the study area at Rishikesh (Dehradun) region.

The following statistics equations adopted to calculate the various bio-statistical parameters of the parasite (Table I) [4].

Incidence of infection (X1) $=\frac{\text { No.of infected hosts (b) }}{\text { No.of examined hosts (a) }}$

Intensity of infection $(\mathrm{X} 2)=\frac{\text { No.of collected parasites(c) }}{\text { No.of infected hosts (b) }}$

Density of infection (Y1) $=\frac{\text { No.of collected parasites (c) }}{\text { No.of examined hosts (a) }}$

Index of infection $(\mathrm{Y} 2)=$

No.of infected hosts (b)) $\times$ (No.of collected parasites (c) No. of examined hosts $\left(a^{2}\right)$

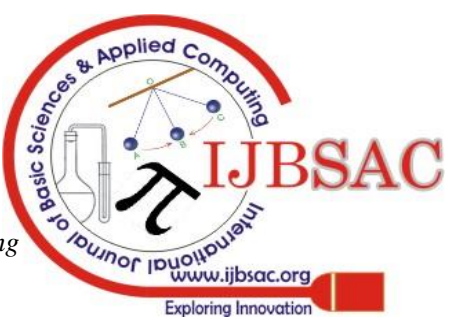


Table- I: Showing statistical analysis of the population of host fishes at sampling stations.

\begin{tabular}{|c|c|c|c|c|c|c|c|c|}
\hline \multicolumn{9}{|c|}{ Sampling station District - Dehradun (Tehsil - Rishikesh) U.K. } \\
\hline $\begin{array}{l}\text { Sr. } \\
\text { No. }\end{array}$ & $\begin{array}{l}\text { Sampling } \\
\text { stations }\end{array}$ & $\begin{array}{l}\text { No. of } \\
\text { examined } \\
\text { hosts } \\
\text { (a) }\end{array}$ & $\begin{array}{l}\text { No. of } \\
\text { infected } \\
\text { hosts } \\
\text { (b) }\end{array}$ & $\begin{array}{c}\text { No. of } \\
\text { collected } \\
\text { parasites } \\
\text { (c) }\end{array}$ & $\begin{array}{c}\text { Incidence } \\
\text { of } \\
\text { infection } \\
\text { (X1) }\end{array}$ & $\begin{array}{c}\text { Intensity } \\
\text { of } \\
\text { infection } \\
\text { (X2) }\end{array}$ & $\begin{array}{c}\text { Density } \\
\text { of } \\
\text { infection } \\
\text { (Y1) }\end{array}$ & $\begin{array}{l}\text { Index of } \\
\text { infection } \\
\text { (Y2) }\end{array}$ \\
\hline 1. & $\begin{array}{c}\text { Birpur } \\
\text { Khurd near } \\
\text { Ganga river } \\
\text { line, } \\
\text { Rishikesh }\end{array}$ & 30 & 6 & 11 & 0.2 & 1.833 & 0.366 & 0.073 \\
\hline 2. & $\begin{array}{c}\text { Bishan } \\
\text { Garh near } \\
\text { Ganga river } \\
\text { line, } \\
\text { Rishikesh }\end{array}$ & 30 & 4 & 7 & 0.133 & 1.75 & 0.233 & 0.031 \\
\hline 3. & $\begin{array}{l}\text { Ram Jhula } \\
\text { near river } \\
\text { Ramganga, } \\
\text { Rishikesh }\end{array}$ & 30 & 5 & 9 & 0.166 & 1.8 & 0.3 & 0.05 \\
\hline 4. & $\begin{array}{c}\text { Fish } \\
\text { reservoirs, } \\
\text { Mal Devta } \\
\text { farm, } \\
\text { Dehradun }\end{array}$ & 30 & 5 & 10 & 0.166 & 2 & 0.333 & 0.055 \\
\hline 5. & $\begin{array}{c}\text { Fish tanks, } \\
\text { Malsi Dear } \\
\text { Park, } \\
\text { Dehradun }\end{array}$ & 30 & 3 & 6 & 0.1 & 2 & 0.2 & 0.02 \\
\hline 6. & $\begin{array}{c}\text { Water } \\
\text { bodies in } \\
\text { Aglar } \\
\text { Valley, hill } \\
\text { town of } \\
\text { Mussoorie }\end{array}$ & 30 & 7 & 13 & 0.233 & 1.857 & 0.433 & 0.101 \\
\hline & Total & 180 & 30 & 56 & 0.998 & 11.24 & 1.865 & 0.33 \\
\hline
\end{tabular}

Table- II: Biometric characteristic (weight and total length) of freshwater host fishes with Prevalence of Cestode/ Nematode/ Trematode/ Acanthocephala parasites.

\begin{tabular}{|c|c|c|c|c|c|c|c|c|c|c|c|}
\hline \multirow{2}{*}{$\begin{array}{c}\text { Sr. No. } \\
\text { (Sampling } \\
\text { stations) }\end{array}$} & \multirow{2}{*}{$\begin{array}{c}\text { No. of } \\
\text { examined } \\
\text { hosts }\end{array}$} & \multirow{2}{*}{$\begin{array}{c}\text { No. of } \\
\text { infected } \\
\text { hosts }\end{array}$} & \multirow[t]{2}{*}{$\begin{array}{l}\text { Host } \\
\text { fishes }\end{array}$} & \multirow[t]{2}{*}{$\begin{array}{c}\text { Infected } \\
\text { site (Habitat) }\end{array}$} & \multirow[t]{2}{*}{$\begin{array}{c}\text { Weight } \\
\text { (gm) }\end{array}$} & \multirow[t]{2}{*}{$\begin{array}{c}\text { Total } \\
\text { Length }(\mathrm{cm})\end{array}$} & \multirow{2}{*}{$\begin{array}{l}\text { No. of } \\
\text { collected } \\
\text { Parasite } \\
\text { s }\end{array}$} & \multicolumn{4}{|c|}{ Parasites } \\
\hline & & & & & & & & C & $\mathbf{N}$ & $\mathbf{T}$ & $\mathbf{A}$ \\
\hline 1. & 30 & 6 & $\begin{array}{l}\text { Clarias } \\
\text { batrachus }\end{array}$ & Intestine & $185-352$ & $12.3-19.6$ & 11 & 1 & 5 & 4 & 1 \\
\hline 2. & 30 & 4 & $\begin{array}{l}\text { Clarias } \\
\text { batrachus }\end{array}$ & Intestine & $189-325$ & $16.5-22.5$ & 7 & 1 & 4 & 2 & 0 \\
\hline 3. & 30 & 5 & $\begin{array}{l}\text { Clarias } \\
\text { batrachus, } \\
\text { Channa } \\
\text { punctatus }\end{array}$ & $\begin{array}{c}\text { Intestine, } \\
\text { Body cavity }\end{array}$ & $240-372$ & $14.5-18.6$ & 9 & 2 & 4 & 2 & 1 \\
\hline 4. & 30 & 5 & $\begin{array}{l}\text { Clarias } \\
\text { batrachus }\end{array}$ & Intestine & $205-342$ & $19.4-31.2$ & 10 & 2 & 4 & 3 & 1 \\
\hline 5. & 30 & 3 & $\begin{array}{l}\text { Clarias } \\
\text { batrachus, } \\
\text { Channa } \\
\text { punctatus }\end{array}$ & $\begin{array}{l}\text { Intestine, } \\
\text { Gills }\end{array}$ & $315-389$ & $14.8-21.6$ & 6 & 1 & 3 & 2 & 0 \\
\hline 6. & 30 & 7 & $\begin{array}{l}\text { Clarias } \\
\text { batrachus }\end{array}$ & $\begin{array}{c}\text { Intestine, } \\
\text { Body cavity }\end{array}$ & $257-412$ & $17.4-23.9$ & 13 & 3 & 5 & 3 & 2 \\
\hline
\end{tabular}


Table- III. Showing morphometric measurement L×W (in mm) and cladistic taxonomical characters of Genus Lytocestus Cohn ${ }^{[17]}$, 1908 tapeworms under generic

\begin{tabular}{|c|c|c|c|c|c|c|c|c|c|c|c|c|c|c|}
\hline $\begin{array}{l}\text { Sr. } \\
\text { No. }\end{array}$ & Characters & $\begin{array}{l}\text { Lytocestus } \\
\text { indicus } \\
\text { (Re-describ } \\
\text { ed) }\end{array}$ & $\begin{array}{l}\text { Lytocestus } \\
\text { osmanaba } \\
\text { densis } \\
\text { (Bhure } \\
\text { et. al., } \\
\text { 2010) }\end{array}$ & $\begin{array}{l}\text { Lytocest } \\
\text { us } \\
\text { marath } \\
\text { w-adens } \\
\text { is } \\
\text { (Shinde } \\
\text { et. al, } \\
\text { 1988) } \\
{ }^{[6]}\end{array}$ & $\begin{array}{l}\text { Lytocestus } \\
\text { follicularae } \\
\text { (Bhure et. } \\
\text { al., } \\
2010)^{[5,7]}\end{array}$ & $\begin{array}{l}\text { Lytocest } \\
\text { us } \\
\text { puranen } \\
\text { sis } \\
\text { (Kasar } \\
\text { et. al., } \\
\text { 2010) }\end{array}$ & $\begin{array}{l}\text { Lytocestu } \\
s \\
\text { clariae } \\
\text { (Tandon } \text { et. al., } \\
\text { 2005) } \\
\text { [9] }\end{array}$ & $\begin{array}{l}\text { Lytocestu } \\
\text { s } \\
\text { longicollis } \\
\text { (Devi PR } \\
\text { et. al., } \\
\text { 1973) }\end{array}$ & \begin{tabular}{|l|} 
Lytocestus \\
parvulus \\
(Kasar et. \\
al., \\
2010) \\
${ }^{[8]}$
\end{tabular} & $\begin{array}{l}\begin{array}{l}\text { Lytocestus } \\
\text { clariae }\end{array} \\
\text { (Re-descri } \\
\text { bed) } \\
\text { (Singh et. } \\
\text { al., } \\
2020)^{[11]}\end{array}$ & 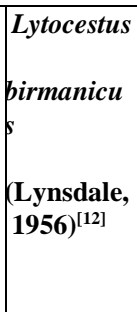 & $\begin{array}{l}\text { Lytocestus } \\
\text { filiformis } \\
\text { (Woodlan } \\
\text { d,1923 } \\
\text { and } \\
\text { Fuhrman } \\
\text { n } \\
\text { et. al., } \\
\text { 1925) }\end{array}$ & $\begin{array}{l}\text { Lytocestu } \\
s \\
\text { attenuatu } \\
s \\
\text { Tandon } \\
\text { et.al., } \\
\text { 2005) }{ }^{[9]}\end{array}$ & $\begin{array}{l}\text { Lytocestus } \\
\text { heteropneu } \\
\text { stii (Tando } \\
\text { n et.al., } \\
\text { 2005) }\end{array}$ \\
\hline 1. & $\begin{array}{l}\begin{array}{l}\text { Length of } \\
\text { the worm }\end{array} \\
\text { the }\end{array}$ & 13 & 11.52 & 12.22 & 12.26 & 14.69 & 12.10 & 11.76 & 12.46 & 12.06 & 11.58 & 12.67 & 13.20 & 11.80 \\
\hline 2. & $\begin{array}{l}\text { Maximum } \\
\text { breadth of } \\
\text { the worm }\end{array}$ & 0.61 & 0.82 & 0.90 & 0.70 & 0.95 & 0.79 & 0.75 & 0.80 & 0.69 & 0.85 & 0.96 & 1.05 & 0.62 \\
\hline 3. & Neck & $0.48 \times 0.55$ & $0.61 \times 0.22$ & $\begin{array}{c}0.53 \times 0 . \\
23\end{array}$ & $0.46 \times 0.18$ & $\begin{array}{l}0.49 \times 0.2 \\
4\end{array}$ & $0.69 \times 0.19$ & $0.58 \times 0.24$ & $0.68 \times 0.19$ & $0.69 \times 0.16$ & $\begin{array}{l}0.62 \times 0.2 \\
0\end{array}$ & $0.50 \times 0.23$ & $0.65 \times 0.31$ & $0.60 \times 0.26$ \\
\hline 4. & $\begin{array}{l}\text { Ovary: } \\
\text { shape }\end{array}$ & $\begin{array}{l}\mathrm{H} \text { and } \\
\text { butterfly } \\
\text { flap shaped }\end{array}$ & H shaped & $\begin{array}{c}\text { Butterfl } \\
\text { y } \\
\text { shaped }\end{array}$ & H shaped & $\begin{array}{l}\text { Inverted } \\
\text { U shaped }\end{array}$ & $\begin{array}{c}\text { Butterfly } \\
\text { shaped }\end{array}$ & $\begin{array}{l}\text { Inverted } \\
\text { A-shaped }\end{array}$ & X shaped & $\begin{array}{l}\text { H shaped } \\
\text { like a } \\
\text { butterfly }\end{array}$ & H shaped & $\begin{array}{l}\text { Inverted U } \\
\text { shaped }\end{array}$ & $\begin{array}{l}\text { Butterfly } \\
\text { shaped }\end{array}$ & $\begin{array}{l}\text { Inverted } \\
\text { A-shaped }\end{array}$ \\
\hline 5. & \begin{tabular}{|l|} 
Genital \\
aperture \\
(pore)
\end{tabular} & Present & Present & Present & Present & Present & Present & Present & Present & Present & Present & Present & Present & Present \\
\hline 6. & $\begin{array}{l}\text { Vitelline } \\
\text { follicles }\end{array}$ & $0.098 \times 0.052$ & $0.6 \times 0.13$ & $\begin{array}{l}0.5 \times 0.1 \\
2\end{array}$ & $0.5 \times 0.12$ & $0.6 \times 0.12$ & $0.7 \times 0.11$ & $0.5 \times 0.9$ & $0.05 \times 0.12$ & $0.05 \times 0.13$ & $0.6 \times 0.12$ & $0.6 \times 0.13$ & $0.4 \times 0.10$ & $0.6 \times 0.9$ \\
\hline 7. & $\begin{array}{l}\text { Cirrus } \\
\text { Pouch }\end{array}$ & $\begin{array}{l}\text { well-develo } \\
\text { ped oval to } \\
\text { round }\end{array}$ & $\begin{array}{l}\text { Round to } \\
\text { oval }\end{array}$ & Round & $\begin{array}{l}\text { Symmetric } \\
\text { al oval }\end{array}$ & $\begin{array}{l}\text { Round to } \\
\text { oval }\end{array}$ & $\begin{array}{l}\text { Oval to } \\
\text { round }\end{array}$ & $\begin{array}{l}\text { Mostly } \\
\text { oval }\end{array}$ & $\begin{array}{l}\text { Round to } \\
\text { oval }\end{array}$ & $\begin{array}{l}\text { Well } \\
\text { developed, } \\
\text { oval to } \\
\text { round }\end{array}$ & $\begin{array}{l}\text { Round to } \\
\text { oval }\end{array}$ & $\begin{array}{l}\text { Round to } \\
\text { oval }\end{array}$ & Round & Mostly oval \\
\hline 8. & Eggs & $0.037 \times 0.022$ & $0.04 \times 0.02$ & $\begin{array}{c}0.04 \times 0 . \\
01\end{array}$ & $0.05 \times 0.03$ & $\begin{array}{l}0.04 \times 0.0 \\
3\end{array}$ & $0.05 \times 0.02$ & $0.05 \times 0.02$ & $0.06 \times 0.03$ & $0.05 \times 0.02$ & $\begin{array}{l}0.04 \times 0.0 \\
2\end{array}$ & $0.04 \times 0.04$ & $0.05 \times 0.01$ & $0.05 \times 0.03$ \\
\hline 9. & \begin{tabular}{|l}
$\begin{array}{l}\text { Excretory } \\
\text { pore }\end{array}$ \\
\end{tabular} & $0.016 \times 0.035$ & $0.03 \times 0.07$ & $\begin{array}{l}0.04 \times 0 . \\
7\end{array}$ & $0.03 \times 0.06$ & $\begin{array}{l}0.05 \times 0.0 \\
8\end{array}$ & $0.03 \times 0.06$ & $0.05 \times 0.08$ & \begin{tabular}{|l|l}
$0.04 \times 0.9$ \\
\end{tabular} & $0.03 \times 0.08$ & $\begin{array}{l}0.03 \times 0.0 \\
8\end{array}$ & $0.05 \times 0.09$ & $0.04 \times 0.7$ & $0.05 \times 0.08$ \\
\hline
\end{tabular}

Retrieval Number: L01770421220/2020@BEIESP DOI:10.35940/ijbsac.L0177.0321120 Journal Website: www.ijbsac.org
Published By: Blue Eyes Intelligence Engineering \& Sciences Publication

10 (c) Copyright: All rights reserved.

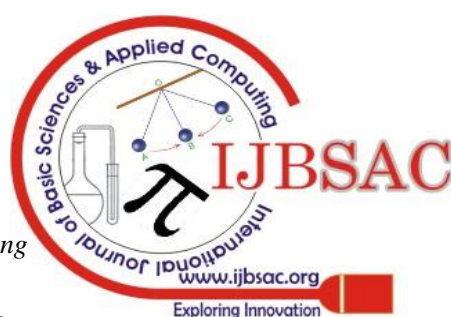




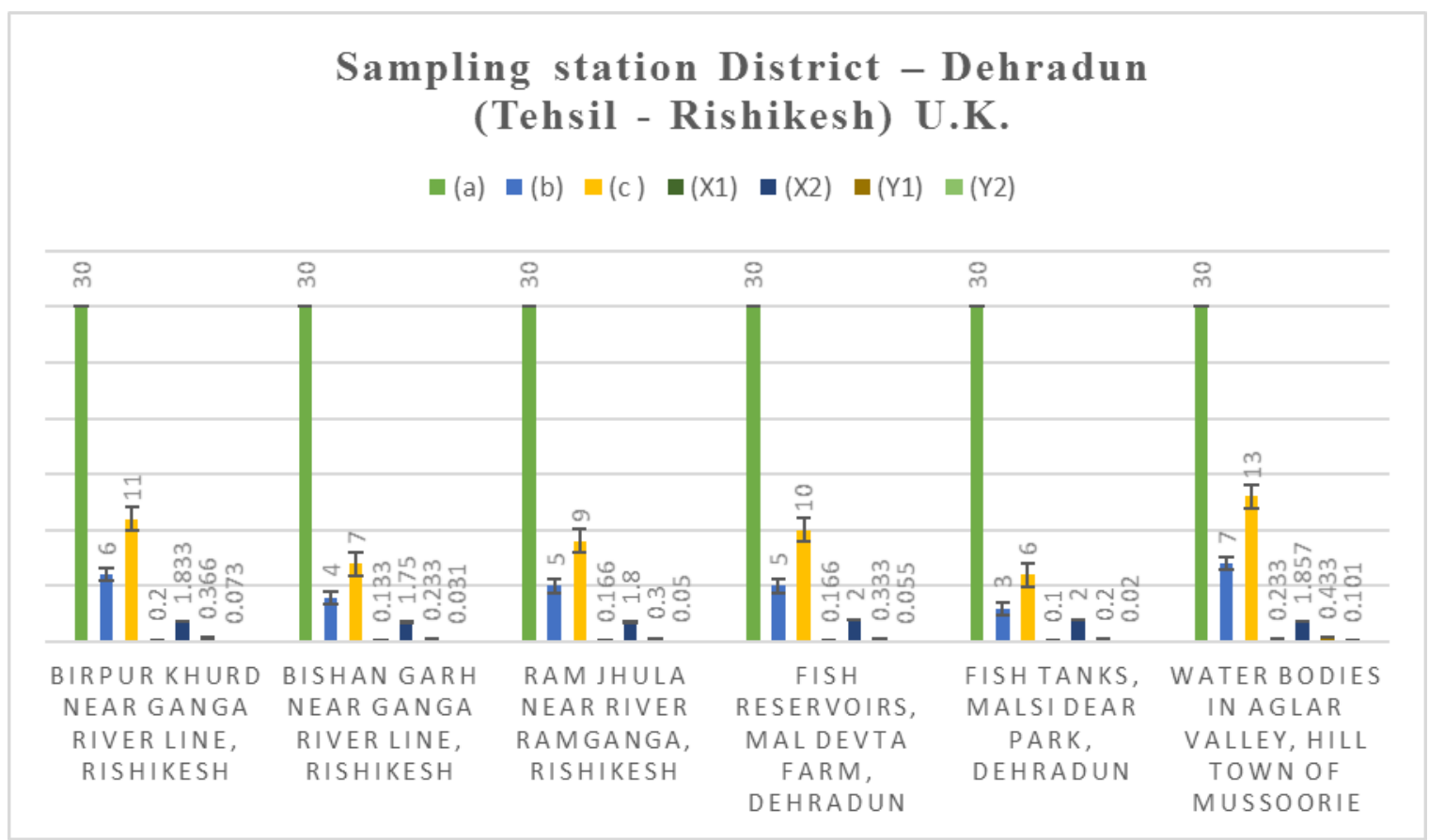

Fig. 3. Graphical presentation of sampling stations.

A detailed account of rare tapeworm parasite of freshwater catfish (Clarias batrachus Linnaeus, 1758) ${ }^{[3]}$ is described. Shape, size, morpho-micrometry, infection site, etc. are observed under a compound microscope and camera lucida figures are drawn (Fig.1, Table II). All morphometrical measurements are measured in millimeters using a stage micrometer and an ocular micrometer (Table III).

Tapeworms measure $12-14 \times 0.35-0.87(13 \times 0.61)$. Scolex is medium-sized, flat, smooth, blunt without any major groove. Narrow at the top measures $0.22-0.45 \times 0.35-0.53$ $(0.33 \times 0.44)$. Neck present with measures 0.43-0.53×0.48-0.62 $(0.48 \times 0.55)$. Proglottids absent. Numerous testes are present in the medullary region, numerable, oval to round shaped Measures $0.05-0.14 \times 0.07-0.09(0.09 \times 0.11)$. Vas deferens are visible. Vitelline Follicle present in the cortical and medullary region measures $0.090-0.106 \times 0.045-0.060(0.098 \times 0.052)$. Some VF surrounds touches the ovarian lobes. Uterus extends the posterior region to the ovary. Uterus long, non-glandular, coiled medullary, situated posterior and anterior to the ovarian isthmus. Cirrus pouch well-developed oval to round with internal seminal vesicle. Median measures 0.04-0.05 $\times 0.03-0.02(0.04 \times 0.02)$. Genital aperture present. Ovary $\mathrm{H}$ shaped like a butterfly flap, both arms even, lateral lobes of ovary situated in cortical and medullary regions measures $0.45-0.43 \times 0.39-0.27(0.42 \times 0.33)$ behind the cirrus pouch. Genital pore present at the posterior region of the worm. Isthmus present and curved in the medullary region. Anterior uterus measures 0.03-0.04×0.12-0.14 $(0.03 \times 0.13)$. Excretory pore measures $0.015-0.018 \times 0.030-0.040$ $(0.016 \times 0.035)$. Eggs are some oval and bilobed, broader than length, some coffee bean-like shape and operculate structure measures $0.045-0.030 \times 0.030-0.015(0.037 \times 0.022)$.

\section{RESULT AND DISCUSSION}

This parasite species comes closer to genera Lytocestus. This species differs from Pseudolytocestus in having

well-developed scolex, external seminal vesicle absent, internal seminal vesicle present, ovary $\mathrm{H}$ shaped like butterfly and uterus extend up to post ovarian region [15].

1) The present form differs from Lytocestus osmanabadensis in having a well-developed Scolex with neck, ovary $\mathrm{H}$ shaped, and bilobed eggs [5,7].

2) The present form differs from Lytocestus marathwadensis in having medium size worm with well-developed scolex, $\mathrm{H}$ shaped ovary, uterus extends up to post ovarian region and bilobed, operculated eggs [6].

3) The Present form differs from Lytocestus follicularae having mediums size of worm, $\mathrm{H}$ butterfly-shaped ovary in absence of Mehlis gland [7].

4) The present form differs from Lytocestus puranensis in having a well-developed Scolex with neck, internal seminal vesicle present, and $\mathrm{H}$ shaped ovary [8].

5) This form differs from Lytocestus clariae in having medium-sized worms, presence of neck (under segmented), presence of internal seminal vesicle and butterfly-shaped ovary [9].

6) The present form differs from Lytocestus longicollis in having internal seminal vesicle, $\mathrm{H}$ shaped ovary and bilobed eggs [10].

7) The present form differs from Lytocestus parvulus in having an $\mathrm{H}$ shaped ovary and bilobed, operculate eggs [8].

8) The present form differs from Lytocestus birmanicus in having butterfly flap shaped ovary, longer than broader eggs [12].

9) The present form differs from Lytocestus filiformis in having $\mathrm{H}$ shaped ovary, round to oval eggs, Internal seminal vesicle present [13]. 
Taxonomy of Piscian Cestodes of Freshwater fish: A cladistic model of Morphotaxonomic Study of Rare Tapeworm from the Dehradun Region (U.K.), India

10) The present form differs from Lytocestus attenuates in having long Isthmus, butterfly-shaped ovary, Internal seminal vesicle present, broader than longer ovular eggs [9].

11) The present form differs from Lytocestus heteropneustii in having a well-developed ovary, uterus, internal seminal vesicle, excretory pore [9].

On the basis of taxonomic comparison with morphometrical characters present tapeworm holds a specific classified hierarchy in the Lytocestidae family.

\begin{tabular}{|c|c|}
\hline \multicolumn{2}{|c|}{ Taxonomic Summary } \\
\hline Kingdom & Animalia \\
\hline Phylum & Platyhelminthes \\
\hline Class & Cestoda \\
\hline Order & Caryophyllidea \\
\hline Family & $\begin{array}{l}\text { Lytocestidae Wardle et } \\
\text { McLeod, 1952[16] }\end{array}$ \\
\hline Genus & 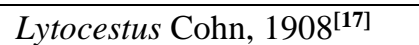 \\
\hline Species & indicus (Redescribed) \\
\hline Host & $\begin{array}{l}\text { Claris batrachus (Linn. } \\
1758)^{[3]}\end{array}$ \\
\hline Habitat & Intestine \\
\hline Locality & $\begin{array}{l}\text { Ganga riverside at Dehradun } \\
\text { district, Rishikesh tehsil } \\
\text { (U.K.) }\end{array}$ \\
\hline $\begin{array}{l}\text { Number of } \\
\text { Parasites } \\
\end{array}$ & $06 / 10$ \\
\hline $\begin{array}{l}\text { NCBI Taxonomy } \\
\text { ID }\end{array}$ & 647077 \\
\hline ITIS TSN & 1060037 \\
\hline $\begin{array}{l}\text { Global } \\
\text { Biodiversity } \\
\text { Information } \\
\text { Facility ID }\end{array}$ & 7010074 \\
\hline IRMNG ID & 11469553 \\
\hline
\end{tabular}

\section{CONCLUSION}

The Lytocestus indicus is considered as uncommon Caryophyllidea cestode of Clarias batrachus in terms of cladistic morphotaxonomy. The morphological demographic diversity of its population has observed in listed sampling stations of the Dehradun region. The anatomical structure and morphotaxonomic studies are particularly needed to prevent cestode parasite infection in edible fishes. In case of heavy infestation of cestode parasites in host fishes, it is observed as serious injuries in intestine, gills and body mass by worm's scolex and hooks penetration. To get more information about cestode and observing the cladistic morphotaxonomy and morphometry this type of research is necessary.

\section{ACKNOWLEDGMENT}

The authors are thankful to the Head, Department of Zoology, Institute of Basic Science, Bundelkhand University Campus, Jhansi (U.P.) for providing the laboratory facilities and valuable suggestions.

3. Case studies on fish loss assessment of small-scale fisheries in Indonesia, FIAM/C1129 (En), FAO Fisheries and Aquaculture Circular, ISSN 2070-6065.

4. C. Linnaeus, Systema Naturae per regna tria naturae, secundum classes, ordines, genera, species, cum characteribus, differentiis, synonymis, locis. Editio Decima, Reformata. Tomus I Laurentii salvii Stockholm 1758, 824 pp. DOI: 10.5962/bhl.title.542 bhl

5. L. Margolis, G. W. Esch, J. C. Holmes, A. M. Kuris and G. A. Schad, "The use of ecological terms in parasitology" (report of an ad hoc committee of the American Society of Parasitologists). Journal of Parasitology 1982, 68 pp. 131-133.

6. D. B. Bhure, S. B. Waghmare, C. R. Kasar and K. M. Shaikh, "Taxonomic Observation of the Caryophyllidea tapeworm Lytocestus Cohn, 1908 from Clarias batrachus (Linneus, 1758)". Journal of Ecology and Environmental Sciences 2010, 1(1) pp. 01-06.

7. G. B. Shinde and A. N. Phad, "On a new Cestode Lytocestus marathwadensis from freshwater fish". Riv Di Para 1988, 47(2) pp. 295-298.

8. D. B. Bhure and S. S. Nanware, "Faunestic Diversity and Distribution of Cestode Parasites of Piscean, Avian and Mammalian Host". American Journal of Biology and Life Sciences 2015, 3(6) pp. 241-245 (http://www.openscienceonline.com/journal/ajbls)

9. C. R. Kasar, D. B. Bhure, S. S. Nanware and M. B. Sonune, "New species of the caryophyllidean tapeworm Lytocestus Cohn, 1908 from Clarius batrachus (Linneus,1758)". The Asian Journal of Animal Science 2010, Vol. 5(2) pp. 219-222.

10. V. Tandon, R. Chakravarty and B. Das, "Four new species of the genus Lytocestus (Caryophyllidea, Lytocestidae) from Edible Catfishes in Assam and Meghalaya, India”. Journal of Parasitic Diseases 2005, Vol. 29(2) pp. 131.

11. P. R. Devi, "Lytocestus longicollis sp. nov. (Cestoidea: Caryophyllidea) from catfish Clarias batrachus (L.) in India”. Journal of Helminthology 1973, Vol. 47 pp. 415-420.

12. A. J. Singh, A. Narayan and S. Singh, "Morphotaxonomic Cladistic Analysis of Rare Cestode Parasites from the Prayagraj Region (U.P.), India". International Journal of Basic Sciences and Applied Computing (IJBSAC) 2020 ISSN: 2394-367X, Volume-2 Issue-10, pp 16-19.

13. J. A. Lynsdale, "On two new species of Lytocestus from Burma and the Sudan respectively”. J Helminthol. 1956, 30(2-3) pp. 87-96.

14. W. N. F. Woodland, "On some remarkable new forms of Caryophyllaeidae from the Anglo-Egyptian Sudan, and a revision of the families of the Cestodaria”. Q J Microsc Sci (New Series) 1923, Vol. 67 pp. 435-472.

15. O. Fuhrmann, J. G. Baer, "Zoological results of the third Tanganyika expedition conducted by Dr. W. A. Cunnington, 1904-1905”. Report on the Cestodes. In: Proceedings of the Zoological Society of London 1925, pp. 79-100.

16. A. Pathak and A. K. Srivastav, "Morphotaxonomical study of a new cestode, Pseudoclariasis pandei n.g., n.sp from catfish, Clarias batrachus Linn.," Flora and Fauna 2015, Vol. 21(1) pp. 15-20, ISSN 09716920.

17. R. A. Wardle and J. A. McLeod, The Zoology of tapeworms. University of Minnesota Press, Minneapolis 1952, pp. 780.

18. L. Cohn, Die Anatomic eines neuen Fischcestoden. Centralblatt fur Bakteiologie, Parasitenkunde, Infection-shrank-heiten und Hygiene. Abteilung L Originale 1908; Vol. 46 pp. 134-139.

\section{AUTHORS PROFILE}

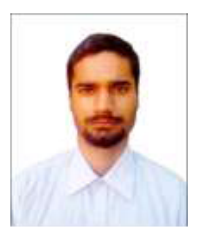

Amar Jeet Singh, Research Scholar (Zoology) Parasitology Laboratory, Department of Zoology, Institute of Basic Science, Bundelkhand University Campus, Jhansi (U.P.) India. Present working project: Taxonomy of piscian cestodes of fresh water fish. Specialization: Parasitology \& Immunology Email amarjeet7321@gmail.com

Blue Eyes Intelligence Engineering \& Sciences Publication

$$
\begin{aligned}
& \text { \& Sciences Publication } \\
& \text { (C) Copyright: All rights reserved. }
\end{aligned}
$$




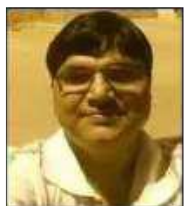

Aditya Narayan, Assistant Professor, Department of

Zoology, Institute of Basic Science, Bundelkhand

University, Jhansi (U.P.) India. Email

adityanarayan21@gmail.com

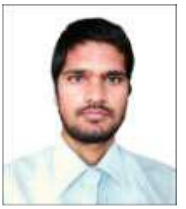

Sarvjeet Singh, Research Scholar (NST) Department of NST, Science Block, DTU, Delhi Email sarvjeetsingh779@gmail.com 